\title{
Targeted inhibition of myeloid-derived suppressor cells by doxorubicin to enhance antigen-specific cytotoxic T lymphocytes killing neuroblastoma cells in vitro
}

\author{
Weili $\mathrm{Xu}^{1}$, Suolin $\mathrm{Li}^{1}$, Fengxue $\mathrm{Yu}^{1}$, Meng $\mathrm{Li}^{1}$, Hui Zhou ${ }^{1}$, and Xiaofeng Yang ${ }^{1}$ \\ ${ }^{1}$ Second Hospital of Hebei Medical University
}

July 1, 2020

\begin{abstract}
Background High agglomeration of myeloid-derived suppressor cell (MDSC) in neuroblastoma (NB) resulted in immune tolerance and impeded therapeutic effects. Doxorubicin (DOX) is currently found the most specific drug to selectively remove MDSC. However, whether these mechanisms can relieve the inhibitory role of MDSC in NB immunotherapy is still remain unclear. Procedure In this study, the inhibitory role of MDSC for NB Ag-specific cytotoxic T lymphocyte (CTL) were investigated in vitro. CTLs, NB cells, MDSCs and DOX were mixed and cultivated in different collocation pattern, the levels of cluster

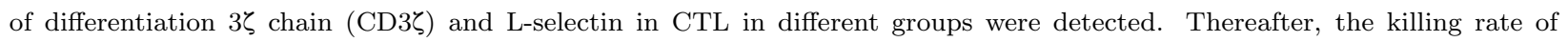
neuroblastoma cells, secretion of interleukin-2 and interferon- $\gamma$ were detected and compared. Results The proliferation and

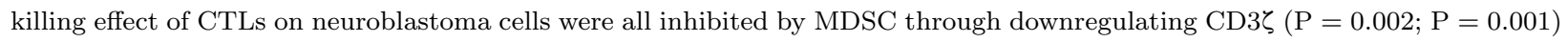
and L-selectin $(\mathrm{P}=0.006 ; \mathrm{P}<0.001)$ by reai-time $\mathrm{PCR}$ test and by Western-blot analysis respectively. However, this inhibitory role can be effectively reversed by doxorubicin. The significant difference existed in the killing rate between the groups $(\mathrm{P}<$ 0.001) except between CTL+SK-N-SH group and CTL+SK-N-SH+DOX group $(\mathrm{P}>0.05)$. There were significant differences in the levels of IL-2 $(\mathrm{P}<0.001)$ and IFN- $\gamma(\mathrm{P}<0.001)$ among the groups. Conclusions This study provided the novel method to enhance immunotherapeutic effects for neuroblastoma by using doxorubicin to targeted inhibition of MDSCs.
\end{abstract}

\section{Introduction}

Neuroblastoma (NB) is the most common pediatric neuroendocrine tumors with high malignancy and early metastasis. The survival rate of children with high-risk NB is still less than $40 \%$ although many therapeutic method including surgery, radiotherapy, chemotherapy and stem cell transplantation have been adopted. So, a new therapeutic method is urgently needed to raise curative effect and reduce the recurrence and metastasis for $\mathrm{NB}{ }^{1,2}$. Although immunotherapy in children with solid tumors started lately, many studies have shown that immunotherapy of NB can effectively remove the tumor cells and reduce tumor recurrence and metastasis with higher specificity and less toxicity compared with the traditional therapy ${ }^{3}$.

However, in the survival microenvironment of NB, all kinds of complicated immunosuppressive factors impede therapeutic effects and result in immune tolerance, including downregulation of HLA-I antigen and accumulation of various immunosuppressive cells such as myeloid-derived suppressor cell (MDSC), regulatory T cell and tumor associated macrophage, etc ${ }^{4-8}$. Currently, new approaches targeting the tumor microenvironment hold promise for further improvements in survival and long-term quality of life ${ }^{9}$. Furthermore, Jales A found high expression of disialoganglioside in surface of NB resulted in increasing of MDSC agglomeration in tumor microenvironment ${ }^{10}$, which triggered tumor immunosuppression and promoted the development and metastasis of tumor. So, in these factors, MDSC may be the main impediment to NB immunotherapy. Some studies described MDSCs caused dissociation between T-cell receptor (TCR) and cluster of differentiation $3 \zeta(\mathrm{CD} 3 \zeta)$ molecules, disrupting TCR complexes on $\mathrm{T}$ cells which result in Ag-specific $\mathrm{CD} 8^{+} \mathrm{T}$ 
cell tolerance in cancer ${ }^{11}$. MDSC down-regulate L-selectin levels on naive T cells, decreasing their ability to home to sites where they would be activated ${ }^{12}$. Alizadeh D et al pointed out doxorubicin (DOX) can be used as a potent immunomodulatory agent that selectively impair MDSC-induced immunosuppression in breast cancer ${ }^{13}$. However, whether these mechanisms can explain the inhibitory role of MDSC in NB immunotherapy is still remain unclear.

Therefore, in the present study, we proposed BALB/c mice as the experimental object, successfully prepared NB Ag-specific CTLs, and grouping mixed cultivation of CTL, neuroblastoma cells, MDSC and DOX. Thereafter, the killing rates of CTL to neuroblastoma cells, secretion of Interleukin-2 (IL-2) and interferon- $\gamma$

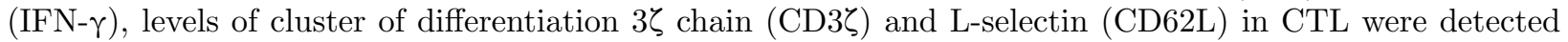
and compared in different groups respectively in order to explore mechanism of immune tolerance caused by MDSC in NB and provide the new method to enhance the immune therapeutic effects of NB by using doxorubicin to targeted inhibition of myeloid-derived suppressor cells.

\section{Materials and methods}

Cell lines and animals

SK-N-SH cells, obtained from Shanghai Cell Bank of Chinese Academy of Medical Sciences, were maintained in DMEM (Gibco) with $10 \%$ fetal calf serum (Gibco), and cultivated at $37^{\circ} \mathrm{C}$ in a $5 \% \mathrm{CO}_{2}$ humidified incubator. 6 week female BALB/c mice, each weighing 18 to $20 \mathrm{~g}$ (SCXK Hebei 2008-1-003), were purchased from the Experimental Animal Center of Hebei Province (SPF grade, SYXK Hebei 2008-0026) and housed in a specific pathogen-free facility. Spirit, diet and defecation of mice were regularly observed and recorded. All experiments were conducted in accordance with the principles and procedures outlined in the Guideline of Institutional Experimental Animal Review Committee.

Separation and purification of MDSC in vitro

BALB/c mice were sacrificed, then bone marrow cells were extracted and made into single cell suspension. After red blood cells having been cracked, cell suspension was administrated by anti-Gr-1 monoclonal $\mathrm{Ab}(\mathrm{mAb})$, anti-CD11b mAb, anti-CD80 mAb, anti-F4/80 mAb, anti-CD11c mAb and anti-MHCII mAb. Differentiation of MDSC and proportion of $\mathrm{Gr}-1^{+} \mathrm{CD} 11 \mathrm{~b}^{+}$MDSCs were detected by flow cytometry.

Bone marrow cells were suspended by PBS and MDSC were separated by Percoll Density Gradient Centrifugation. Then, cell suspension was collected and separated by CD11b magnetic bead and purity of Gr$1^{+} \mathrm{CD} 11 \mathrm{~b}^{+} \mathrm{MDSC}$ was detected by flow cytometry.

Preparation of neuroblastoma antigen-specific cytotoxic T lymphocyte (CTL)

Extraction and cultivation of $B A L B / c$ mice bone marrow derived dendritic cell (DC)

$\mathrm{BALB} / \mathrm{c}$ mice were sacrificed, then bone marrow cells were extracted and made into single cell suspension. After red blood cells having been cracked, cell suspension was cultivated according to $10^{6} / \mathrm{ml}$ concentration. Thereafter, rmGM-CSF $(20 \mathrm{ng} / \mathrm{ml})$ and rmIL-4 $(20 \mathrm{ng} / \mathrm{ml})$ were added. On the sixth day, morphology of DCs was observed by phase contrast microscope and the expressive rate of CD11c, CD86 and MHC-II were detected by flow cytometry.

Tumor antigen loading DC

SK-N-SH cells in logarithmic phase were resuspended and adjusted to $2 \times 10^{7} / \mathrm{ml}$ concentration. Cells were quickly frozen to -80 , then rewarmed in 37 water. After four cycles, cells suspension was centrifuged with $10,000 \mathrm{r} / \mathrm{min}$ for 15 minutes and the supernatant was collected and cryopreserved at -80 . The lysate equivalent to $2 \times 10^{6}$ tumor cells was added into per millilite rmedium of DCs and cultivated for 6 days. Four hours later, cells suspension was added rmTNF- $\alpha$ and cultivated to 7th day. Then, the suspension cells were collected and defined tumor antigen loaded DCs. The cell morphology of DCs was observed by phase contrast microscope. Meanwhile, the expression rate of CD11c, CD86 and MHC-II were detected by flow cytometry. 
Extraction, identification and cultivation of $\mathrm{CD} 3^{+} \mathrm{T}$ cells

$\mathrm{CD} 3^{+} \mathrm{T}$ cells were extracted from mice spleen lymphocytes suspension by Mouse CD3 ${ }^{+}$Tcell Magnetic Bead. Magnetic-activated cell sorting (MACS) can be repeated for several times and about $3 \times 10^{6} \mathrm{~T}$ lymphocytes can be extracted from each mouse spleen. The purified lymphocytes were collected and identified purity of $\mathrm{CD}^{+} \mathrm{T}$ cells. Concentration of cells was adjusted to $5 \times 10^{6} / \mathrm{ml}$ and cultivated 2 days in medium containing rmIL-2 $(20 \mathrm{ng} / \mathrm{ml})$. Then, cell morphology was observed under the phase contrast microscope and cell vitality was tested by Trypan blue.

Preparation of SK-N-SH Ag-specific CTL and cytokine release test

After $\mathrm{CD} 3^{+} \mathrm{T}$ cells were cultivated for 2 days, DCs loaded tumor antigen were added according to the proportion of DC:T = 1:20. At the same time, rmIL-2 $(20 \mathrm{ng} / \mathrm{ml})$ was mixed and cultivated for 3 or 4 days. Thereafter, morphology of $\mathrm{T}$ cells was observed under inverted microscope. After $\mathrm{T}$ cells had been activated for 72 hours, the supernatants in each group were retained and concentrations of IL-2 and IFN- $\gamma$ were calculated respectively according to the absorbance value and the standard curve measured by enzyme standard instrument. IL-2 and IFN- $\gamma$ in the supernatant between the different groups were compared.

MDSC inhibiting proliferation of neuroblastoma antigen-specific CTL

The activated CTLs were stained by 5,6-carboxyfluorescein diacetate succinimidyl ester (CFSE) (final concentration of CFSE was $5 \mu \mathrm{mol} / \mathrm{L}^{14}$ ) and divided into two groups. In one group, CTLs were mixed with Gr- $1^{+}$CD11b ${ }^{+}$MDSC (4:1) separated by MACS and stimulated proliferation by CD3/CD28 antibody. Meanwhile, in another group, all of the things was same except absence of MDSC. A parallel control group was set up for each group with the same method as the experimental group. After being cultivated for 4 days, the mixed cell system were observed by fluorescence microscopy and detected cell proliferation by flow cytometry.

Detection of CD3ろ and CD62L in CTL by real-time PCR and Western-blot

CTL, CTL+MDSC(1:4) and CTL+MDSC(1:4)+DOX(2umol/L) were incubated respectively. The contents of CD3 $\zeta$ and CD62L in CTL of the different groups were assessed and compared by real-time PCR. Total RNA in samples was isolated using the ultra-pure RNA extraction kit according to the manufacturer's guidelines (Cwbio, Co. Ltd.). For reverse transcription, $1 \mu \mathrm{g}$ of RNA was used to synthesize single-strand cDNA (HiFi-MMLV cDNA first strand synthesis kit; Cwbio, Co. Ltd.) according to the manufacturer's guidelines. Real-time PCR was performed using a fluorescence quantitative PCR amplifier (LightCycler 480 II, Roche Inc., Germany), as described elsewhere. Triplicate reactions were set up for each gene in a 96-well plate. Reaction information is summarized in Table 1. Amplification was performed with the following program: initial denaturation at $95^{\circ} \mathrm{C}$ for $15 \mathrm{~min}$, followed by 40 cycles of $95^{\circ} \mathrm{C}$ for $10 \mathrm{~s}, 58^{\circ} \mathrm{C}$ for $30 \mathrm{~s}$ and $72^{\circ} \mathrm{C}$ for $30 \mathrm{~s}$. The experimental data were expressed as relative expression using the $2^{-\Delta \Delta^{\circ} \tau}$ equation as described previously ${ }^{15}$.

Proteins were extracted with RIPA lysis buffer (Invitrogen) and quantitatively detected with the BCA Protein Assay kit (Invitrogen). The samples were separated by $12 \%$ SDS-PAGE, transferred to a PVDF membrane, and blocked by $5 \%$ BSA. The primary antibodies against each protein were added and incubated at $4^{\circ} \mathrm{C}$ overnight. Afterward, the second antibodies were added and incubated for $4 \mathrm{~h}$ at $20^{\circ} \mathrm{C}$. After washing the membrane, chemiluminescence was detected on X-ray film by FluorChem®HD2 (Alpha Innotech, Santa Clara, CA, USA), with GAPDH as the internal reference.

MDSC regulating the killing effect of neuroblastoma antigen-specific CTL in vitro

CTL, CTL+MDSC (1:4), and CTL+MDSC(1:4)+DOX $(2 \mu \mathrm{mol} / \mathrm{L})$ were mixed with SK-N-SH cells and incubated respectively (CTL:SK-N-SH $=20: 1$ ). At the same time, blank control group, target cells group and effector cells group were set up. CCK- 8 was added into the cell system (20 $\mu$ l/hole) after incubation and absorbance value (A value) was detected in $450 \mathrm{~nm}$ wavelength by enzyme standard instrument. The cytotoxic activities of effector cells were evaluated by the killing rate. The killing rate of effector cells was calculated as follow, kill rate $(\%)=[$ A value of target cells group - (A value of experimental group - A value 
of effector cells group)]/A value of target cells group $\times 100 \%$. The killing rates of CTLs to SK-N-SH cells between the groups were compared. The secretion levels of IL-2 and IFN- $\gamma$ in the supernatant between the groups were detected and compared by enzyme-linked immunosorbent assay (ELISA).

Statistical analysis

All data were analyzed using the SAS software (ver. 8 for Windows; SAS Institute Inc., NC, USA). The Wilcoxon two-sample test and a repeated-measures analysis of variance were used to analyze the results. $P$ $¡ 0.05$ was deemed to indicate statistical significance.

\section{Results}

MDSC separation, extraction, identification and neuroblastoma Ag-specific CTL preparation, cytokine release test

By percoll density gradient centrifugation, the rate of $\mathrm{Gr}-1^{+} \mathrm{MDSC}, \mathrm{CD} 11 \mathrm{~b}^{+} \mathrm{MDSC}, \mathrm{CD} 11 \mathrm{c}^{+} \mathrm{MDSC}$, CD80 ${ }^{+} \mathrm{MDSC}, \mathrm{F} 4 / 80^{+} \mathrm{MDSC}$ and $\mathrm{MHC}^{-\mathrm{II}^{+} \mathrm{MDSC}}$ were $70.4 \%, 3.5 \%, 4.8 \%, 1.2 \%, 0.3 \%, 2.1 \%$ respectively (Fig. 1A), and the rate of $\mathrm{Gr}-1^{+} \mathrm{CD} 11 \mathrm{~b}^{+} \mathrm{MDSC}$ was $22.6 \%$ (Fig. 1B). Furthermore, MDSC suspension was sorted by CD11b magnetic bead and purification of $\mathrm{Gr}-1^{+} \mathrm{CD} 11 \mathrm{~b}^{+} \mathrm{MDSC}$ was shown $84.6 \%$ by flow cytometry (Fig. 1C). By flow cytometry, the expressive rates of CD11c, CD86 and MHC-II on dendritic cells (DCs) wituout antigen-loaded were $10.9 \%, 3.8 \%$ and $27.9 \%$ respectively which suggested their weaker antigen presenting ability (Fig. 1D). Most of DCs can be seen adherent growth with different size, star or spindle shape and stretching tubers. At the 7th day, DCs were activated by tumor antigen. DCs in half adherent state increased obviously with radial spike and bigger shape. The expressive rates of CD11c, CD86 and MHC-II were $74.8 \%, 50.3 \%$ and $49.8 \%$ respectively which indicated mature DCs with efficient presenting antigen ability (fig. 1E). CD $3^{+} \mathrm{T}$ cells, extracted from spleen lymphocytes by MACS, reached $87.3 \%$ by flow cytometry. The living cells rate was $96.38 \%$ by Trypan blue test. After 3 to 4 days' cultivation with antigenloaded DCs, antigen-specific CD $3^{+} \mathrm{T}$ cells were prepared and gathered into many small colonies. The levels of interleukin-2 (IL-2) $(1.092+/-0.010 \mathrm{ng} / \mathrm{l})$ and interferon- $\gamma(0.855+/-0.038 \mathrm{ng} / \mathrm{l})$ in the supernatant of antigen-loaded $\mathrm{CD}^{+} \mathrm{T}$ cells were significantly higher than IL-2 $(0.962+/-0.007 \mathrm{ng} / \mathrm{l})$ and IFN- $\gamma(0.765+/-$ $0.010 \mathrm{ng} / \mathrm{l})$ in the supernatant of $\mathrm{CD}^{+} \mathrm{T}$ cells without antigen-loaded $(P<0.05)$.

MDSC inhibiting proliferation of CTL

Under fluorescence microscopy, the number of CTLs was same in the two groups before cultivation (Fig. 2A). However, after having been cultivated for 4 days, cells proliferated obviously but staining intensity weakened in CTL group. On the contrary, in CTL+MDSC group, cell number scarcely increased and staining intensity still remain strong (Fige. 2B).

Analysis of flow cytometry showed consistent results. In CTL+MDSC group, CTLs with strong fluorescence $(87.6 \%)$ were much more than CTLs with weak fluorescence. However, strong fluorescence CTLs (44.1\%) were less than weak fluorescence CTLs in CTL group (Fig. 2C).

Downregulating levels of CD3ל and CD62L in CTL by MDSC

Respectively, the levels of CD3 $\zeta$ and CD62L in CTL were detected and compared by reai-time PCR (Fig.


$P=0.002<0.05)$ and CD62L $(F=13.858, P=0.006<0.05)$ occurred between CTL group, CTL + MDSC group and CTL+MDSC+DOX group. Western-blot analysis showed the same results. Significant difference of CD3 $(F=28.241, P=0.001<0.05)$ and CD62L $(F=41.142, P<0.001)$ were also seen among the three groups.

Doxorubicin inhibits MDSC then enhances the killing effect of CTL in vitro

Under inverted microscope, the killing process of CTLs to SK-N-SH cells was clearly shown in each group respectively. From day 1 to day 10, we found that in SK-N-SH+CTL+MDSC groups, SK-N-SH cells 
still scattered in view with relative regular outline. However, in CTL+SK-N-SH group and CTL+SK$\mathrm{N}-\mathrm{SH}+\mathrm{MDSC}+\mathrm{DOX}$ groups, nearly all of SK-N-SH cells appeared apoptosis or necrosis, and CTLs also decreased significantly. During the whole period, in SK-N-SH group, tumor cells proliferated continuously and kept active state (Fig. 4A). So these results proved that doxorubicin inhibits MDSC then enhances the killing effect of CTL on SK-N-SH cells. The significant difference existed in the killing rate between the groups $(F=22.386, P<0.001)$ except between CTL+SK-N-SH group and CTL+SK-N-SH+DOX group $(P=0.100>0.05)$ (Fig. 4B). IL-2 and IFN- $\gamma$ in the supernatant were detected by ELISA. By repeated measurement analysis of variance, the results showed that there were significant differences in the secretion levels of IL-2 $(F=192.013, P=0.000<0.001)$ and IFN- $\gamma(F=519.274, P=0.000<0.001)$ among CTL+SK-N-SH group, CTL+MDSC+SK-N-SH group and CTL+MDSC+SK-N-SH+DOX group (Figs. 4C and $4 \mathrm{D})$.

\section{Discussion}

At present, the most effective method of immunotherapy for NB is adoptive transfer chimeric GD2 antigen receptors CTL ${ }^{6,16}$. However, some phase III clinical trials found that some children with high-risk NB underwent adoptive cell transfer immunotherapy of chimeric GD2 antigen receptors CTL still appeared recurrence and metastasis although the survival rates have improved. This suggested this kind of passive immunotherapy need improve its curative effect by adjusting the immunosuppressive microenvironment ${ }^{4,16}$.

In tumor immune microenvironment, accumulation of a variety of immunosuppressive cells including MDSC formed the main immunosuppressive factors and resulted in immune tolerance and disability of the immune system ${ }^{3}$. MDSC is a group of innate immune cells originated from myeloid which play a negative immune regulative role in tumor progression ${ }^{17,18}$. Some studies suggested MDSC inhibit the body's natural immune by inhibiting DC, accelerating polarization of macrophages to M2, decreasing interleukin-12 and reducing function of NK cells. on the other hand, MDSC inhibit T cells adoptive immunotherapy through high expression of arginase-1, inducible nitric oxide synthase and reactive oxygen species ${ }^{4,19-21}$. Therefore, MDSC induced tumor immune tolerance and became the main impediment to immunotherapy.

The role of MDSC in neuroblastoma still remain unclear although the mechanism of MDSC in other tumors has been explicated too much. In the present study, neuroblastoma Ag-specific CTLs were stained by CFSE. The cytoplasm with fluorescent protein were evenly distributed to the next generation of cells and the fluorescence intensity reduced half when cells proliferated continuously. So, the more generations of cell division, the weaker of cell fluorescence intensity. Therefore, in this study, the fluorescence intensity of CTL became weaker and the number of CTL increased if CTL cultivated without MDSC. Contrarily, cells proliferation decreased and the fluorescence intensity unchanged if CTL cultivated with MDSC. The result fully showed MDSC can inhibit proliferation of neuroblastoma Ag-specific CTL obviously.

In further study, the expressions of $\mathrm{CD} 3 \zeta$ and $\mathrm{CD} 62 \mathrm{~L}$ in Ag-specific CTLs decreased significantly when CTL cultivated with MDSC, but the two proteins raised again when DOX administration. As a chain of

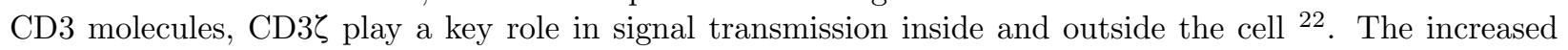
expressive activity of $\zeta$ chain will promote TCR identified immune signal transmission to intracellular which lead to activation of T cells and produce amounts of cytokines, such as IFN- $\gamma$, IL2, etc ${ }^{23}$. Moreover, CD62L is an important molecule involving the extravasation of lymphocytes from the blood and lymphatics, and their homing to lymph nodes and tumors ${ }^{24-26}$. Therefore, the results indicated DOX could targeted relieve


migration of Ag-specific CTL and effectively kill neuroblastoma cells.

In recent years, some researches have pointed out CTL produce cytotoxicity to target cells by two separate ways, namely Perforin way and PCD way mediated by Fas antigen molecules ${ }^{27-29}$. Of them, Perforin way was a major way and played a significant role in antiviral, intracellular bacteria, tumor and immune pathology, etc ${ }^{29-31}$. During the course of Perforin way, CTL killed tumor meanwhile the cytokines IL-2 and IFN- $\gamma$ were released. The more obvious of the killing effect, the higher concentrations of the cytokine, and vice versa. In the present study, the killing rate of NB cells and the levels of IL-2 and IFN- $\gamma$ in supernatant 
were all decreased significantly when CTLs cultivated with MDSC. However, this inhibitory role of MDSC can be effectively reversed by DOX administration. Some researches pointed out doxorubicin can selectively eliminates MDSCs and promots the activity of immune effector cells and improves the therapeutic profile of adoptively transferred helper $\mathrm{T}$ lymphocytes ${ }^{13,32,33}$. So, in this study, we demonstrated the underlying mechanism of targeting inhibition of myeloid-derived suppressor cells by doxorubicin to enhance antigenspecific cytotoxic $\mathrm{T}$ lymphocytes killing neuroblastoma cells in vitro.

\section{Conflicts of interest}

All authors have declared no financial conflicts regarding this work.

\section{Acknowledgments}

This study was supported by the National Natural Science Foundation of China (No.81472503). We thank Huizhen GENG, Jie ZHANG and Luping LI for their technical assistance and the development facility for their help in sorting the dendritic cells and myeloid-derived suppressor cellmyeloid cells. We also thank Prof. Zhen-yun Mou (Department of Statistics Research Office, Hebei Medical University) for his help with statistical analysis.

\section{References}

1. Yuantao HOU, Lu LIU, Changlin WANG. Advances in immunotherapy of neuroblastoma. Clinical J Pediatric Surg. 2011; 10(4): 290-293. doi: 10.3969/j.issn.1671-6353.2011.04.020

2. Heczey A, Louis CU. Advances in chimeric antigen receptor immunotherapy for neuroblastoma. Discov Med. 2013; 16(90): 287-294. PMCID: PMC4106238

3. Seeger RC. Immunology and Immunotherapy of Neuroblastoma. Semin Cancer Biol. 2011; 21(4): 229-237. doi: 10.1016/j.semcancer.2011.09.012.

4. Pistoia V, Morandi F, Bianchi G, Pezzolo A, Prigione I, Raffaghello L. Immunosuppressive microenvironment in neuroblastoma. Front Oncol. 2013; 3: 167(1-8). doi: 10.3389/fonc.2013.00167.

5. Tilak T, Sherawat S, Agarwala S, Gupta R, Vishnubhatla S, Bakhshi S. Circulating T-regulatory cells in neuroblastoma: a pilot prospective study. Pediatr Hematol Oncol. 2014; 31(8): 717-722. doi: 10.3109/08880018.2014.886002.

6. Long AH, Highfill SL, Cui Y, Smith JP, Walker AJ, Ramakrishna S, El-Etriby R, Galli S, Tsokos MG, Orentas RJ, et al. Reduction of MDSCs with all-trans retinoic acid improves CAR therapy efficacy for sarcomas. Cancer Immunol Res. 2016; 4(10): 869-880. doi: 10.1158/2326-6066.CIR-15-0230.

7. Komohara Y, Takeya M. CAFs and TAMs: maestros of the tumour microenvironment. J Pathol. 2017; 241(3): 313-315. doi: 10.1002/path.4824.

8. Hashimoto O, Yoshida M, Koma Y, Yanai T, Hasegawa D, Kosaka Y, Nishimura N, Yokozaki H. Collaboration of cancer-associated fibroblasts and tumour-associated macrophages forneuroblastoma development. J Pathol. 2016; 240(2): 211-223. doi: 10.1002/path.4769.

9. Matthay KK, Maris JM, Schleiermacher G, Nakagawara A, Mackall CL, Diller L, Weiss WA. Neuroblastoma. Nat Rev Dis Primers. 2016; 2: 16078. doi: 10.1038/nrdp.2016.78.

10. Jales A, Falahati R, Mari E, Stemmy EJ, Shen W, Southammakosane C, Herzog D, Ladisch S, Leitenberg D. Ganglioside-exposed dendritic cells inhibit T-cell effector function by promoting regulatory cell activity. Immunology. 2011; 132(1): 134-143. doi: 10.1111/j.1365-2567.2010.03348.x.

11. Nagaraj S, Schrum AG, Cho HI, Celis E, Gabrilovich DI. Mechanism of T cell tolerance induced by myeloid-derived suppressor cells. J Immunol. 2010; 184(6): 3106-3116. doi: 10.4049/jimmunol.0902661.

12. Hanson EM, Clements VK, Sinha P, Ilkovitch D, Ostrand-Rosenberg S. Myeloid-derived suppressor cells down-regulate L-selectin expression on CD4+ and CD8+ Tcells. J Immunol. 2009; 183(2): 937944. doi: 10.4049/jimmunol.0804253.

13. Alizadeh D, Trad M, Hanke NT, Larmonier CB, Janikashvili N, Bonnotte B, Katsanis E, Larmonier N. Doxorubicin eliminates myeloid-derived suppressor cells and enhances the efficacy of adoptive T-cell transfer in breast cancer. Cancer Res. 2014; 74(1): 104-118. doi: 10.1158/0008-5472.CAN-13-1545. 
14. Xu W, Cai J, Li S, Zhang H, Han J, Wen M, Wen J, Gao F. Improving the in vivo persistence, distribution and function of cytotoxic $\mathrm{T}$ lymphocytes by inhibiting the tumor immunosuppressive microenvironment. Scand J Immunol. 2013; 78(1): 50-60. doi: 10.1111/sji.12065.

15. Schmittgen TD, Livak KJ. Analyzing real-time PCR data by the comparative $\mathrm{C}(\mathrm{T})$ method. Nat Protoc 2008; 3: 1101-08. PMID: 18546601.

16. Cheung NK, Dyer MA. Neuroblastoma: developmental biology, cancer genomics and immunotherapy. Nat Rev Cancer. 2013; 13(6): 397-411. doi: 10.1038/nrc3526.

17. Gabrilovich DI, Ostrand-Rosenberg S, Bronte V. Coordinate dregulation of myeloid cells by tumors. Nat Rev Immunol. 2012; 12(4): 253-268. doi: 10.1038/nri3175.

18. Jordan KR, Kapoor P, Spongberg E, Tobin RP, Gao D, Borges VF, McCarter MD. Immunosuppressive myeloid-derived suppressor cells are increased in splenocytes from cancer patients. Cancer Immunol Immunother. 2017; 66(4): 503-513. doi: 10.1007/s00262-016-1953-z.

19. Solito S, Pinton L, Mandruzzato S. In Brief: Myeloid-derived suppressor cells in cancer. J Pathol. 2017 Jan 18. [Epub ahead of print] doi: 10.1002/path.4876.

20. Gabrilovich DI. Myeloid-Derived suppressor cells. Cancer Immunol Res. 2017; 5(1): 3-8. doi: 10.1158/2326-6066.CIR-16-0297.

21. Chen J, Ye Y, Liu P, Yu W, Wei F, Li H, Yu J. Suppression of T cells by myeloid-derived suppressor cells in cancer. Hum Immunol. 2017; 78(2): 113-119. doi: 10.1016/j.humimm.2016.12.001.

22. Weiss A, Littman DR. Signal transduction by lymphocyte antigen receptors. Cell. 1994; 76(2): 263-274. PMID: 8293463.

23. Selleri C, Maciejewski JP, Catalano L, Ricci P, Andretta C, Luciano L, Rotoli B. Effects of cyclosporine on hematopoietic and immune functions in patients with hypoplastic myelodysplasia: in vitro and in vivo studies. Cancer. 2002; 95(9): 1911-1922. doi: 10.1002/cncr.10915

24. Hanson EM, Clements VK, Sinha P, Ilkovitch D, Ostrand-Rosenberg S. Myeloid-derived suppressor cells down-regulate L-selectin expression on CD4+ and CD8+ Tcells. J Immunol. 2009; 183(2): 937944. doi: $10.4049 /$ jimmunol.0804253.

25. Ostrand-Rosenberg S. Myeloid-derived suppressor cells: more mechanisms for inhibiting antitumor immunity. Cancer Immunol Immunother. 2010; 59(10): 1593-1600. doi: 10.1007/s00262-010-0855-8.

26. Ku AW, Muhitch JB, Powers CA, Diehl M, Kim M, Fisher DT, Sharda AP, Clements VK, O'Loughlin K, Minderman H, Messmer MN, Ma J, Skitzki JJ, Steeber DA, Walcheck B, Ostrand-Rosenberg S, Abrams SI, Evans SS. Tumor-induced MDSC act via remote control to inhibit L-selectin-dependent adaptive immunity in lymph nodes. Elife. 2016; 5. pii: e17375. doi: 10.7554/eLife.17375.

27. Hassin D, Garber OG, Meiraz A, Schiffenbauer YS, Berke G. Cytotoxic T lymphocyte perforin and Fas ligand working in concert even when Fas ligand lytic action is still not detectable. Immunology. 2011; 133(2): 190-196. doi:10.1111/j.1365-2567.2011.03426.x

28. Kim JH, Kang TH, Noh KH, Bae HC, Kim SH, Yoo YD, Seong SY, Kim TW. Enhancement of dendritic cell-based vaccine potency by anti-apoptotic siRNAs targeting key pro-apoptotic proteins in cytotoxic CD8(+) T cell-mediated cell death. Immunol Lett. 2009; 122(1): 58-67. doi:10.1016/j.imlet.2008.12.006

29. Martínez-Lostao L, Anel A, Pardo J. How do cytotoxic lymphocytes kill cancer cells? Clin Cancer Res. 2015; 21(22):5047-5056. doi: 10.1158/1078-0432.CCR-15-0685.

30. Voskoboinik I, Whisstock JC, Trapani JA. Perforin and granzymes: function, dysfunction and human pathology. Nat Rev Immunol. 2015; 15(6): 388-400. doi:10.1038/nri3839

31. Cullen SP, Brunet M, Martin SJ. Granzymes in cancer and immunity. Cell Death Differ. 2010; 17(4): 616-623. doi:10.1038/cdd.2009.206.

32. Alizadeh D, Katsanis E, Larmonier N. Chemotherapeutic targeting of myeloid-derived suppressor cells. Oncoimmunology. 2014; 3(1): e27359. PMID: 24653963.

33. Hsu FT, Chen TC, Chuang HY, Chang YF, Hwang JJ. Enhancement of adoptive T cell transfer with single low dose pretreatment of doxorubicin or paclitaxel in mice. Oncotarget. 2015; 6(42): 44134-44150. doi: 10.18632/oncotarget.6628.

Figure Legends 
Figure1 Extraction, identification, purification of myeloid-derived suppressor cell (MDSC) and cultivation and tumor antigen loading of dendritic cell (DC) A, Cells were extracted from bone marrow of BALB/c mice and stained by monoclonal antibodies. By flow cytometry, the expressive rate of Gr$1^{+}$MDSC, CD11b ${ }^{+}$MDSC, CD11 $\mathrm{c}^{+}$MDSC, CD $80^{+}$MDSC, F4 $/ 80^{+}$MDSC and MHC-II ${ }^{+}$MDSC were $70.4 \%$, $3.5 \%, 4.8 \%, 1.2 \%, 0.3 \%, 2.1 \%$ respectively. $\mathbf{B}$, The expressive rate of $\mathrm{Gr}-1^{+} \mathrm{CD} 11 \mathrm{~b}^{+} \mathrm{MDSC}$ was $22.6 \% . \mathbf{C}$, After Magnetic-activated cell sorting (MACS) by CD11b magnetic bead, purification of Gr-1 ${ }^{+}$CD11b ${ }^{+}$MDSC reached $84.6 \%$.D, Most of dendritic cells wituout antigen-loaded can be seen adherent growth with different size, star or spindle shape and stretching tubers, but part of the cells seemed half adherent state with rough surface. The expressive rates of CD11c, CD86 and MHC-II on DCs were $10.9 \%, 3.8 \%$ and $27.9 \%$ respectively by flow cytometry.E, At the 7th day, DCs were stimulated and activated by tumor antigen. DCs in half adherent state increased obviously with radial spike and bigger shape. The expressive rates of CD11c, CD86 and MHC-II were $74.8 \%, 50.3 \%$ and $49.8 \%$ respectively by flow cytometry.

Figure2 Myeloid-derived suppressor cell (MDSC) inhibiting proliferation of neuroblastoma antigen-specific cytotoxic T lymphocyte (CTL) Neuroblastoma antigen-specific CTLs in the two groups were stained by 5,6-carboxyfluorescein diacetate succinimidyl ester (CFSE).A, Under fluorescence microscopy, the same number of CTLs was seen in CTL group and CTL+MDSC group before cultivation. B,After having been cultivated for 4 days, cell proliferated obviously but fluorescent intensity weakened in CTL group. However, in CTL+MDSC group, cell fluorescent intensity remain strong and cell number scarcely increased. C, By flow cytometry, the results showed the consistent results with the view in microscopy. After cultivation, In CTL+MDSC group, the rate of CTLs with strong fluorescence was $87.6 \%$. However, only $44.1 \%$ of CTLs with strong fluorescence were found in CTL group.

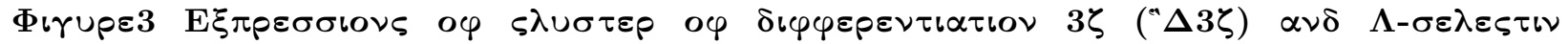


compared by reai-time PCR and western-blot analysis respectively. A, In reai-time PCR test, significant difference of $\mathrm{CD} 3 \zeta(F=20.315, P=0.002<0.05)$ and CD62L $(F=13.858, P=0.006<0.05)$ occurred between CTL group, CTL+MDSC group and CTL+MDSC+DOX group. B, Western-blot analysis showed


were seen among the three groups. ${ }^{*} P\left\lceil 0.05 ;{ }^{* *} P\lceil 0.01\right.$.

Figure4 Immunosuppressive action of MDSC on neuroblastoma antigen-specific CTL and doxorubicin inhibits MDSC then enhances the killing effect of CTL in vitro Under inverted microscope, the killing process of CTLs to SK-N-SH cells was clearly shown in each group respectively. A , As the control group, In SK-N-SH group, tumor cells proliferated continuously and kept active state. At d1, CTLs and SK-N-SH cells were mixed and no obvious difference was seen between the groups. At d2, in CTL+SK-N-SH group and CTL+SK-N-SH+MDSC+DOX group, CTLs and SK-N-SH cells gathered and began to interact. But CTLs and SK-N-SH cells scattered in view in CTL+SK-N-SH+MDSC group. At d4, in CTL+SK-N-SH+MDSC group, CTLs and SK-N-SH cells all proliferated slowly. However, in the other two groups, CTL proliferated but SK-N-SH cells began to deform. At d7, in CTL+SK-N-SH group and CTL+SK-N-SH+MDSC+DOX group, SK-N-SH cells furtherly deformed and lost their cellular shape meanwhile CTLs began to decrease. However, SK-N-SH cells kept regular shape in CTL+SK-N-SH+MDSC group. At d10, in SK-N-SH+CTL+MDSC groups, SK-N-SH cells still scattered in view with relative regular outline. However, in the other two groups, nearly all of SK-N-SH cells appeared apoptosis or necrosis, and CTLs also decreased significantly.B, The significant difference existed in the killing rate between the groups $(F=22.386, P<0.001)$ except between $\mathrm{CTL}+\mathrm{SK}-\mathrm{N}-\mathrm{SH}$ group and $\mathrm{CTL}+\mathrm{SK}-\mathrm{N}-\mathrm{SH}+\mathrm{DOX}$ group $(P=$ $0.100>0.05)$. $\mathbf{C}$ and $\mathbf{D}$, Interleukin-2 (IL-2) and interferon- $\gamma(\mathrm{IFN}-\gamma)$ in the supernatant were detected by ELISA. By repeated measurement analysis of variance, the results showed that there were significant differences in the secretion levels of IL-2 $(F=192.013, P=0.000<0.001)$ and IFN- $\gamma(F=519.274, P=0.000$ $<0.001)$ among CTL+SK-N-SH group, CTL+MDSC+SK-N-SH group and CTL+MDSC+SK-N-SH+DOX group. ${ }^{*} P$ ¡0.05.

Hosted file 
Table.docx available at https://authorea.com/users/338268/articles/464308-targetedinhibition-of-myeloid-derived-suppressor-cells-by-doxorubicin-to-enhance-antigenspecific-cytotoxic-t-lymphocytes-killing-neuroblastoma-cells-in-vitro

A Real-time PCR

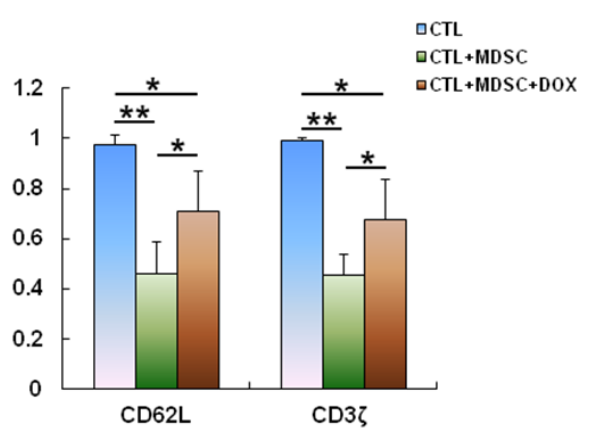

B Western-blot analysis
